\title{
Double-Stranded Helix of Xanthan: Dissociation Behavior in Mixtures of Water and Cadoxen
}

\author{
Takahiro SATO, Takashi NORISUYE, and Hiroshi FUJITA \\ Department of Macromolecular Science, Osaka University, \\ Toyonaka, Osaka 560, Japan \\ (Received October 30, 1984)
}

\begin{abstract}
A sodium salt xanthan sample dissolved directly in mixtures of water and cadoxen was investigated by elastic light scattering and viscometry at $25^{\circ} \mathrm{C}$. With the increase in weight fraction $w_{\text {cad }}$ of cadoxen in the mixed solvent, the weight-average molecular weight, $z$ average radius of gyration $\left\langle S^{2}\right\rangle_{z}^{1 / 2}$, and intrinsic viscosity $[\eta]$ of the sample fairly sharply decreased from the values for the double-helical dimer (in $0.1 \mathrm{M}$ aqueous $\mathrm{NaCl}$ ) to those for the single random coil (in pure cadoxen) in a range of $w_{\text {cad }}$ from 0.3 to 0.8 . It was found that these data can be explained if it is assumed that only these two species coexist in the mixed solvent and if care is taken of the fact, found from measurements on cadoxen solutions diluted with water, that $\left\langle S^{2}\right\rangle_{z}$ and [ $\eta$ ] of the single chain species markedly increase with decreasing $w_{\text {cad }}$ from 1.0 to 0.6. Thus, it was concluded that xanthan double helices in water-cadoxen mixtures dissociate into single chains directly, i.e., without passing through their partial melting. The expansion of the single chain species was attributed to the formation of intramolecular hydrogen bonds.

KEY WORDS Polysaccharide / Xanthan / Double Helix / Dissociation /

Radius of Gyration / Intrinsic Viscosity /
\end{abstract}

Xanthan is an ionic, extracellular polysaccharide produced by the bacterium Xanthomonas campestris; its repeating unit consists of two main chain $\beta$-1,4-D-glucoses and a trisaccharide side chain. ${ }^{1,2}$ Our recent light scattering, viscosity, and sedimentation velocity measurements ${ }^{3-5}$ have led to the finding that the sodium salt of this polysaccharide dissolves as rodlike dimers having a doublestranded helical structure in $0.1 \mathrm{M}$ aqueous sodium chloride $(\mathrm{NaCl})$ and as single random coils in tris(ethylenediamine) cadmium dihydroxide (cadoxen). It was also found ${ }^{4}$ that the intrinsic viscosity $[\eta]$ of $\mathrm{Na}$ salt xanthan in mixtures of water and cadoxen decreases sharply with increasing $w_{\text {cad }}$ (the weight fraction of cadoxen in the mixed solvent) in a range of $w_{\text {cad }}$ from 0.5 to 0.8 .

It appears that this sharp decrease in $[\eta]$ reflects the dissociation of the double helix of xanthan to single coiling chains. However, the viscosity data alone do not tell whether the dissociation proceeds directly or indirectly through intermediate steps including partial unwinding of the helix. Thus, in addition to more viscometric measurements, a light scattering study was undertaken on $\mathrm{Na}$ salt xanthan in water-cadoxen mixtures.

\section{EXPERIMENTAL}

\section{Preparation of Solutions}

The previously investigated $\mathrm{Na}$ salt xanthan sample $^{4}$ X9-3 was used. It was dissolved directly in a water-cadoxen mixture of desired composition at a temperature below $25^{\circ} \mathrm{C}$, and the solution was diluted with the same mixture to different polymer concentrations (see ref 3 for the preparation and chemical composition of our cadoxen). The solutions so prepared were kept standing below $25^{\circ} \mathrm{C}$ for half a day, and then subjected to light scattering and 
viscosity measurements. No appreciable time dependence of scattering intensity and flow time was found at any $w_{\text {cad }}$ studied.

\section{Light Scattering}

Intensity measurements were made on a Fica 50 automatic light scattering photometer with vertically polarized incident light. The apparatus was calibrated in the same way as described previously. ${ }^{3}$ Test solutions with $w_{\text {cad }}>0.4$ were made optically clean by filtration through a Millipore filter (Type FH), and those with $w_{\text {cad }}<0.4$ by the method ${ }^{3}$ employed previously for $0.1 \mathrm{M}$ aqueous $\mathrm{NaCl}$ solutions of $\mathrm{Na}$ salt xanthan.

Specific refractive index increments $(\partial n / \partial c)_{\mu}$ for dialyzed $\mathrm{Na}$ salt xanthan solutions of $w_{\text {cad }}=0.10$ and 0.30 at $25^{\circ} \mathrm{C}$ were determined using a differential refractometer of the modified Schulz-Cantow type, and the data were combined with our previous set of data $^{3}$ for $w_{\text {cad }}>0.5$. Here, the subscript $\mu$ denotes the chemical potentials of diffusible components. The values of $(\partial n / \partial c)_{\mu}$ for given $w_{\text {cad }}$ were interpolated from the combined data.

\section{Viscometry}

Zero shear-rate viscosities of sample $\mathrm{X} 9-3$ in water-cadoxen mixtures at $25^{\circ} \mathrm{C}$ were determined by four-bulb capillary viscometers. The $[\eta]$ data obtained were combined with those determined previously ${ }^{4}$ for the same sample. The values of Huggins' constant were normal $(0.3-0.5)$ at any $w_{\text {cad }}$ studied.

\section{RESULTS AND DISCUSSION}

Composition Dependence of $M_{w}, A_{2},\left\langle S^{2}\right\rangle_{z}^{1 / 2}$, and $[\eta]$

Figures 1 and 2 show, respectively, the concentration dependence of $\left(K c / R_{0}\right)^{1 / 2}$ and the angular dependence of $P(\theta)^{-1}$ for our $\mathrm{Na}$ salt xanthan sample in water-cadoxen mixtures at $25^{\circ} \mathrm{C}$. Here, $K$ is the optical constant, $c$ the polymer mass concentration, $R_{0}$ the reduced scattering intensity at zero scattering angle,

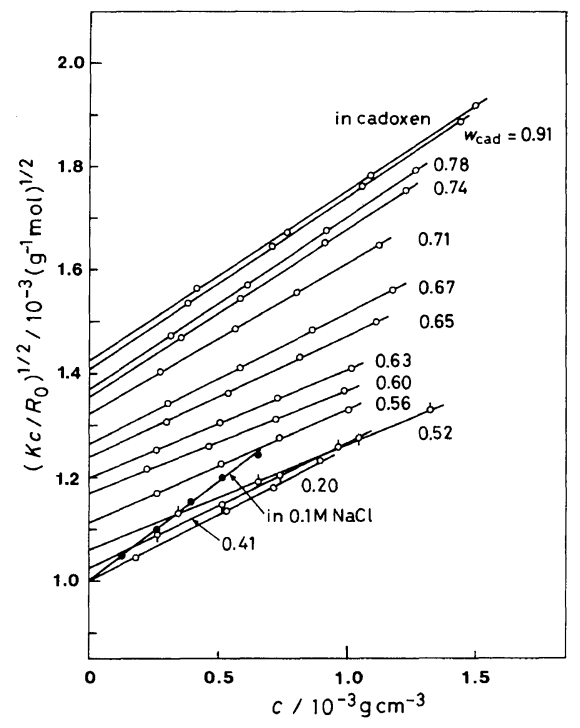

Figure 1. Concentration dependence of $\left(K c / R_{0}\right)^{1 / 2}$ for an $\mathrm{Na}$ salt xanthan sample X9-3 dissolved directly in water-cadoxen mixtures at $25^{\circ} \mathrm{C}$.

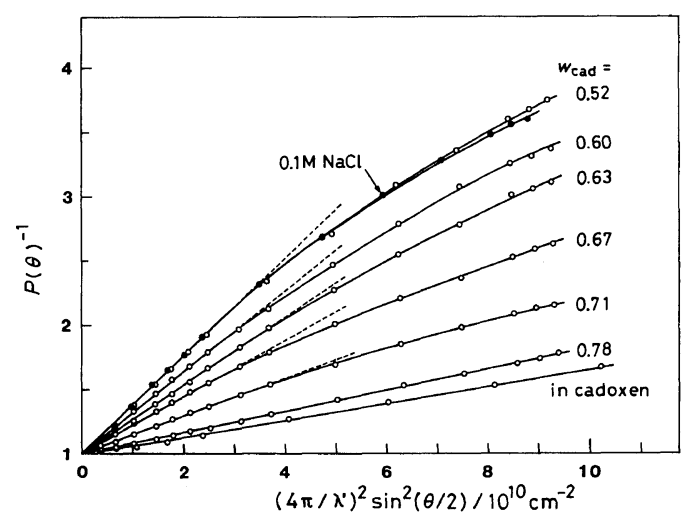

Figure 2. Angular dependence of $P(\theta)^{-1}$ for the xanthan sample dissolved directly in water-cadoxen mixtures at $25^{\circ} \mathrm{C}$. $\lambda^{\prime}$ denotes the wavelength of incident light.

and $P(\theta)$ the particle scattering function.

The values of $M_{w}$ (the weight-average molecular weight), $A_{2}$ (the second virial coefficient), and $\left\langle S^{2}\right\rangle_{z}^{1 / 2}$ (the $z$-average radius of gyration) obtained are plotted against $w_{\text {cad }}$ in Figure 3, along with those of $[\eta]$. The filled circles for $w_{\mathrm{cad}}=0$ in this figure represent the values for the xanthan double helix in $0.1 \mathrm{M}$ aqueous $\mathrm{NaCl}$. Up to $w_{\text {cad }} \sim 0.25, M_{w},\left\langle S^{2}\right\rangle_{z}^{1 / 2}$, and $[\eta]$ do not change much from these val- 
ues, indicating that the double-helical conformation of $\mathrm{Na}$ salt xanthan is maintained in this composition range. As $w_{\text {cad }}$ further increases, $M_{w},\left\langle S^{2}\right\rangle_{z}^{1 / 2}$, and $[\eta]$ all decrease fairly sharply in a range of $w_{\text {cad }}$ from 0.5 to 0.8 and approach the values for the single random coil in pure cadoxen. Interestingly, $A_{2}$ increases sharply in a narrow composition range from 0.6 to 0.8 after passing through a shallow minimum at about $w_{\text {cad }}=0.5$.

\section{Dissociation Behavior}

To analyze the experimental data of Figure 3 , we start with the working hypothesis that xanthan in water-cadoxen mixtures consists only of intact double helices (species 2) and single (separated) chains (species 1) at infinite dilution. Then we can write two relations

$$
\begin{gathered}
\frac{M_{w}\left\langle S^{2}\right\rangle_{z}-M_{1}\left\langle S^{2}\right\rangle_{1}}{M_{2}\left\langle S^{2}\right\rangle_{2}-M_{1}\left\langle S^{2}\right\rangle_{1}}=\frac{M_{w}-M_{1}}{M_{2}-M_{1}} \\
\frac{[\eta]-[\eta]_{1}}{[\eta]_{2}-[\eta]_{1}}=\frac{M_{w}-M_{1}}{M_{2}-M_{1}}
\end{gathered}
$$

which are valid regardless of polydispersity of

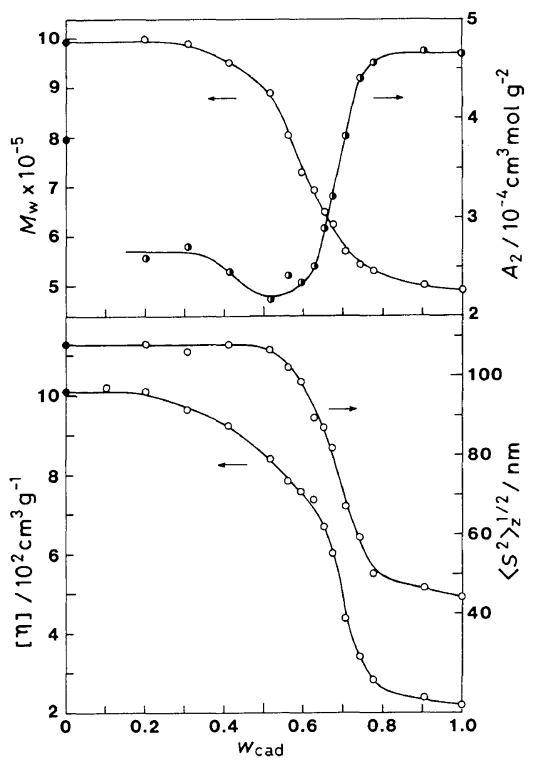

Figure 3. Composition dependence of $M_{w}, A_{2}$, $\left\langle S^{2}\right\rangle_{z}^{1 / 2}$, and $[\eta]$ for the xanthan sample dissolved directly in water-cadoxen mixtures. the sample, and also can substitute for $M_{2}$, $\left\langle S^{2}\right\rangle_{2}$, and $[\eta]_{2}$ the values of $M_{w},\left\langle S^{2}\right\rangle_{z}$, and [ $\eta$ ] measured in $0.1 \mathrm{M}$ aqueous $\mathrm{NaCl}$.

First, the left-hand sides of eq 1 and 2 were evaluated by substituting the values of $M_{w}$, $\left\langle S^{2}\right\rangle_{z}$, and $[\eta]$ in pure cadoxen for $M_{1},\left\langle S^{2}\right\rangle_{1}$, and $[\eta]_{1}$, anticipating that the dimensions of species 1 may little depend on cadoxen composition. The unfilled circles in Figure 4 show the calculated values plotted against $\left(M_{w}-M_{1}\right) /\left(M_{2}-M_{1}\right)$. It can be seen that the points for $\left\langle S^{2}\right\rangle_{z}$ fit the diagonal representing eq 1 , but those for $[\eta]$ markedly deviate upward from the diagonal representing eq 2 for $\left(M_{w}-M_{1}\right) /\left(M_{2}-M_{1}\right)$ between 0.2 and 0.6 . Thus, the data for $[\eta]$ appear not to substantiate our working hypothesis but to sug-

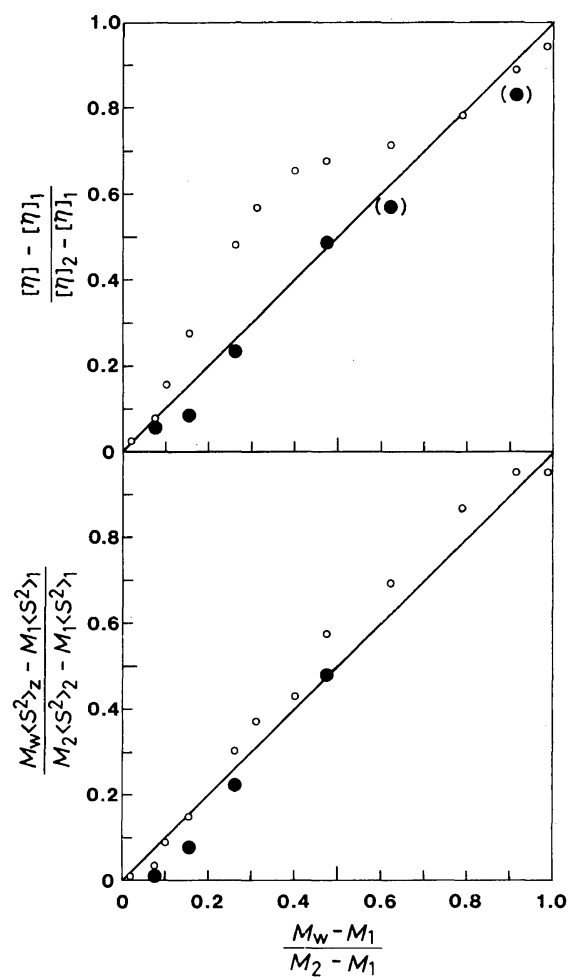

Figure 4. Left-hand side values of eq 1 and 2 plotted against $\left(M_{w}-M_{1}\right) /\left(M_{2}-M_{1}\right)$. Unfilled circles, calculated using the measured $M_{w},\left\langle S^{2}\right\rangle_{z}$, and $[\eta]$ in $0.1 \mathrm{M}$ aqueous $\mathrm{NaCl}$ for $M_{2},\left\langle S^{2}\right\rangle_{2}$, and $[\eta]_{2}$ and those in pure cadoxen for $M_{1},\left\langle S^{2}\right\rangle_{1}$, and $[\eta]_{1}$; filled circles, recalculated using the data of Figure 5 for $\left\langle S^{2}\right\rangle_{1}$ and $[\eta]_{1}$; diagonals, eq 1 and 2 . 
gest more species to coexist with the single chain in the particular range of $w_{\text {cad }}$ corresponding to $\left(M_{w}-M_{1}\right) /\left(M_{2}-M_{1}\right)=0.2-0.6$. Partially melt double helices may be ruled out, since they should lower the points for both $\left\langle S^{2}\right\rangle_{z}$ and $[\eta]$ measurably below the diagonals.

However, we found it unnecessary to hastily abandon our basic hypothesis. This was derived from checking by direct measurement of $\left\langle S^{2}\right\rangle_{1}$ and $[\eta]_{1}$ the relevance of substituting these quantities at $w_{\text {cad }}$ other than unity. The story described below tells the core of our investigation.

It was reported in our previous paper ${ }^{4}$ that the single xanthan chains in cadoxen did not reform double-helical dimers but selfassociated when the solution was diluted with water to $w_{\text {cad }}=0.1$. However, the extent of association was found to be less for the cadoxen solutions of lower xanthan concentrations. This finding suggests that the behavior, e.g., $\left\langle S^{2}\right\rangle_{z}$ and [ $\left.\eta\right]$, of an unassociated xanthan chain at $w_{\text {cad }}=0.10$ can be estimated by preparing a series of water-diluted cadoxen solutions with $w_{\text {cad }}=0.1$ and different polymer concentrations $c$ and extrapolating the data taken on them to $c=0$. Here, a water-diluted cadoxen solution means a solution prepared first by dissolving the polymer in cadoxen and then diluting it with water. By applying the above method to $w_{\text {cad }}$ other than 0.1.we determined $M_{w},\left\langle S^{2}\right\rangle_{z}$, and [ $\left.\eta\right]$ for the one hopefully considered to be an unassociated single xanthan chain, as functions of $w_{\text {cad }}$.

Figure 5 shows the results obtained; see also Figures A1 through A3 in the Appendix. Neither $M_{w}$ nor $\left\langle S^{2}\right\rangle_{z}$ for $w_{\text {cad }}$ below 0.6 was determined with certainty, because the pronounced aggregation of the polymer made the extrapolation to $c=0$ very difficult.

What is significant in Figure 5 is the finding that $M_{w}$ in the composition range of $w_{\text {cad }}$ from 1.0 to 0.6 differs no more than $6 \%$ from that of the single chain in pure cadoxen, whereas both $\left\langle S^{2}\right\rangle_{z}^{1 / 2}$ and $[\eta]$ increase sharply in the same range of $w_{\text {cad }}$. This constancy of $M_{w}$ demonstrates that in water-diluted cadoxen solutions with $w_{\text {cad }} \geq 0.6, \mathrm{Na}$ salt xanthan does not self-associate at infinite dilution and the measured $\left\langle S^{2}\right\rangle_{z}$ and $[\eta]$ can be equated to those for the single chain in a water-cadoxen mixture of given $w_{\text {cad }}(\geq 0.6)$, i.e., $\left\langle S^{2}\right\rangle_{1}$ and $[\eta]_{1}$ in eq 1 and 2 .

The filled circles in Figure 4 represent the left-hand sides of eq 1 and 2 recalculated by using the $\left\langle S^{2}\right\rangle_{z}$ and $[\eta]$ data of Figure 5 for $\left\langle S^{2}\right\rangle_{1}$ and $[\eta]_{1}$. These circles for $[\eta]$ now come closer to the diagonal than the unfilled circles, while those for $\left\langle S^{2}\right\rangle_{z}$ stay as close to the diagonal as the unfilled circles. This result substantiates that our working hypothesis, i.e., the presence of only two species, intact double helix and single chain, need not to be revised at least for $w_{\text {cad }}$ above 0.6. Hence, we conclude that in water-cadoxen mixtures with $w_{\text {cad }}>0.6$, xanthan double helices dissociate into single chains directly, i.e., not passing through intermediate steps including partial unwinding. However, we must wait for future work as regards the situation in mixtures with $w_{\text {cad }}<0.6$.

\section{Interpretation of the Extended Conformation of Single Chains}

The sharp rises in $\left\langle S^{2}\right\rangle_{z}^{1 / 2}$ and $[\eta]$ observed in Figure 5 indicate the extension of single xanthan chains to occur as $w_{\text {cad }}$ is lowered from 0.8 to 0.6 . It is not reasonable to interpret this extension as due to excluded-volume effect, since, as seen from Figure A2, the second virial coefficient for water-diluted cadoxen solutions of xanthan sharply diminishes as $w_{\text {cad }}$ is decreased in this range. Although no data were obtained for $\left\langle S^{2}\right\rangle_{z}$ below $w_{\text {cad }}=0.6$, the observed constancy of $[\eta]$ for $w_{\text {cad }}<0.6$ suggests that the extended single chains keep their dimensions at $w_{\text {cad }}=0.6$ down to very low $w_{\text {cad }}$.

The X-ray analysis of Okuyama et al. ${ }^{6}$ showed that the double-stranded helix (either parallel or antiparallel) of xanthan in the crystalline state has four intramolecular hy- 


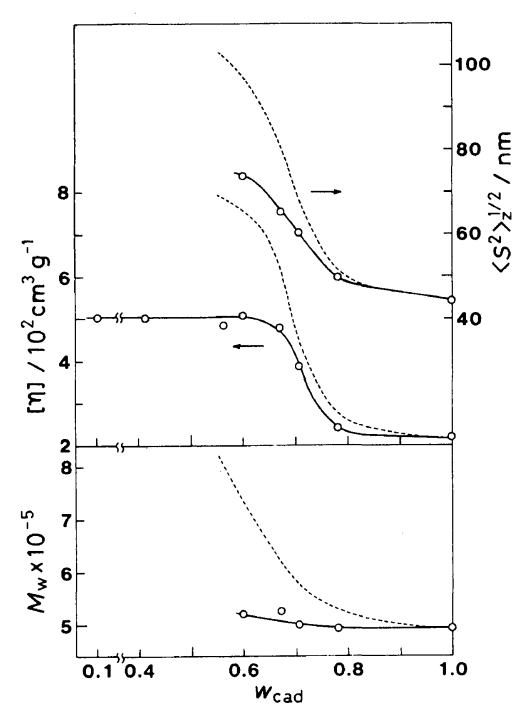

Figure 5. Composition dependence of $M_{w},\left\langle S^{2}\right\rangle_{z}^{1 / 2}$, and $[\eta]$ for the xanthan sample in water-diluted cadoxen solutions (see the text). The dashed lines represent the data in Figure 3.

drogen bonds per repeating unit: one between the $\alpha$-D-mannose and $\beta$-D-glucuronate residues of the side chain, one between a main chain glucose and the $\alpha$-D-mannose attached directly to it, and the other two between the adjacent glucoses in the main chain. The helical conformation of each chain in the double helix is maintained substantially by these hydrogen bonds. ${ }^{6}$

In $\mathrm{Na}$ salt xanthan dissolved directly in water-cadoxen mixtures, the intramolecular hydrogen bonds should remain intact below $w_{\text {cad }} \sim 0.25$, since, as mentioned above, the polysaccharide maintains the double-helical structure in this $w_{\text {cad }}$ range. As cadoxen is added to increase $w_{\text {cad }}$ above 0.25 , these hydrogen bonds should be successively broken and eventually disappear at $w_{\text {cad }}=1$. However, they may be reformed when water is added to a cadoxen solution of a very low xanthan concentration, and more reformation may take place as more water is added. The composition dependence of $\left\langle S^{2}\right\rangle_{z}^{1 / 2}$ and $[\eta]$ in Figure 5 may be explained in terms of this hydrogen bond reformation as follows.

When reformed between adjacent main chain glucoses in a water-diluted cadoxen, the intramolecular hydrogen bonds almost completely hinder the internal rotation about the glucosidic oxygens between the hydrogen bonded glucose rings. This should stiffen the chain, leading to an increase in chain dimensions or intrinsic viscosity. As more hydrogen bonds are reformed at a lower $w_{\text {cad }}$, more glucosidic linkages are fixed. Thus, the chain dimensions should continue to increase with a decrease in $w_{\text {cad }}$ and level off when all possible intramolecular hydrogen bonding* is completed and the internal rotation of every other glucosidic linkages ceases to be possible. The value of $w_{\text {cad }}$ corresponding to this leveling-off is estimated to be 0.6 from the $[\eta]$ data in Figure 5. Though stiffened, the chain below this critical value consists of virtual bonds only about twice as long as that of the randomly coiled chain in pure cadoxen, and hence is still essentially flexible.

At finite polymer concentrations, $\mathrm{Na}$ salt xanthan in water-diluted cadoxen with $w_{\text {cad }}$ below 0.7 was found to self-associate (see Figures A1 and A2 and also ref 4). This process should involve both intramolecular and intermolecular hydrogen bonding. The former must occur much faster than the latter unless the polymer concentration is too high. Hence, it is likely that, in water-diluted cadoxen solutions of finite xanthan concentrations (Figures $\mathrm{A} 1$ and $\mathrm{A} 2$ ), individual chains are stiffened by intramolecular hydrogen bonding before they undergo association by intermolecular hydrogen bonding.

\section{CONCLUDING REMARKS}

The stopped-flow polarimetric measure-

* Intramolecular hydrogen bonds may also be formed between residues distant from each other along the chain contour. However, since this causes the chain to shrink ${ }^{7}$ in contrast to our experimental finding, the fraction of such hydrogen bonds relative to those mentioned in the text may be very small. 
ments of Norton et al. ${ }^{8,9}$ showed that single, disordered xanthan chains in pure water at high temperature became intramolecularly ordered in as short a timescale as about $10 \mathrm{~s}$ when potassium chloride $(\mathrm{KCl})$ was added to the solution. From this finding these authors concluded that xanthan in aqueous salt solutions is dispersed as a single-stranded helix. According to our previous finding, ${ }^{4}$ the double helix of native xanthan in water is destroyed to single chains at elevated temperature, and those chains do not reform double helical dimers when $\mathrm{NaCl}$ is added to the solution and the temperature is lowered to $25^{\circ} \mathrm{C}$. It seems certain that Norton et al.'s experiment was concerned with such single chains and hence found no evidence for the formation of double helices. Thus, we consider their conclusion on a single-stranded helix reasonable and not contradicting ours ${ }^{3-5}$ that native xanthan in aqueous $\mathrm{NaCl}$ is double helical.

Actually, Norton et al.'s samples may be compared with our single chain samples in water-diluted cadoxen solutions. We conjecture that their finding of the intramolecular ordering caused by the addition of $\mathrm{KCl}$ has something to do with our finding of the chain extension caused by the dilution of a cadoxen solution with water. Norton et al. ${ }^{9}$ reported that the intramolecularly ordered single chains or single helices in aqueous $\mathrm{KCl}$ intermolecularly aggregated in a timescale of about $200 \mathrm{~s}$. Interestingly, as mentioned above, the single chains in water-diluted cadoxen undergo pronounced self-association when the polymer concentration is finite.

Acknowledgment. We acknowledge with thanks Grants-in-Aid for Scientific Research and Cooperative Research from the Ministry of Education, Japan.

\section{APPENDIX}

This appendix presents light scattering and viscosity data for water-diluted cadoxen so-

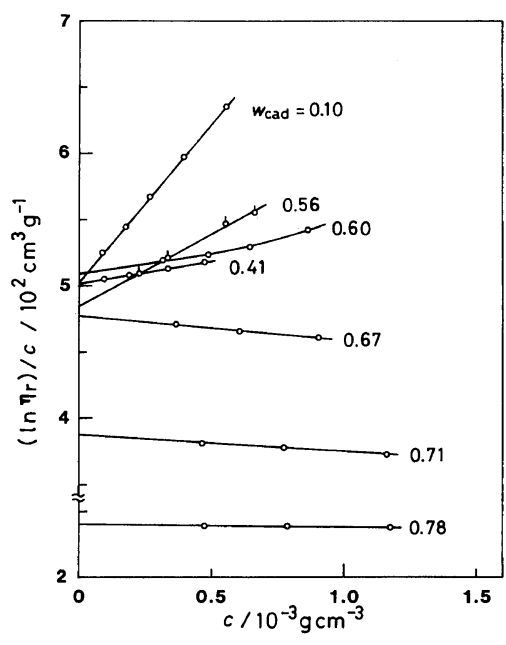

Figure A1. Plots of $\left(\ln \eta_{\mathrm{r}}\right) / c$ vs. $c$ for water-diluted cadoxen solutions of sample X9-3 at $25^{\circ} \mathrm{C}$.

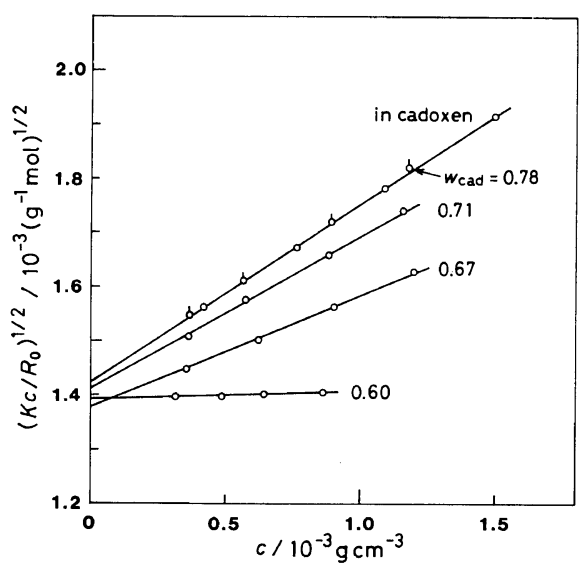

Figure A2. Concentration dependence of $\left(K c / R_{0}\right)^{1 / 2}$ for the xanthan sample in water-diluted cadoxen with different $w_{\text {cad }}$ at $25^{\circ} \mathrm{C}$.

lutions of $\mathrm{Na}$ salt xanthan sample X9-3. Test solutions were prepared by dissolving the sample in pure cadoxen at different $c$, followed by dilution with pure water or a water-cadoxen mixture of appropriate composition to desired $w_{\text {cad }}$. These solutions were subjected to physical measurements half a day after the preparation. The measured scattering intensities and flow times showed no systematic variation with time. This is consistent with the previous finding ${ }^{4}$ that the relative viscosity $\eta_{\mathrm{r}}$ of a 


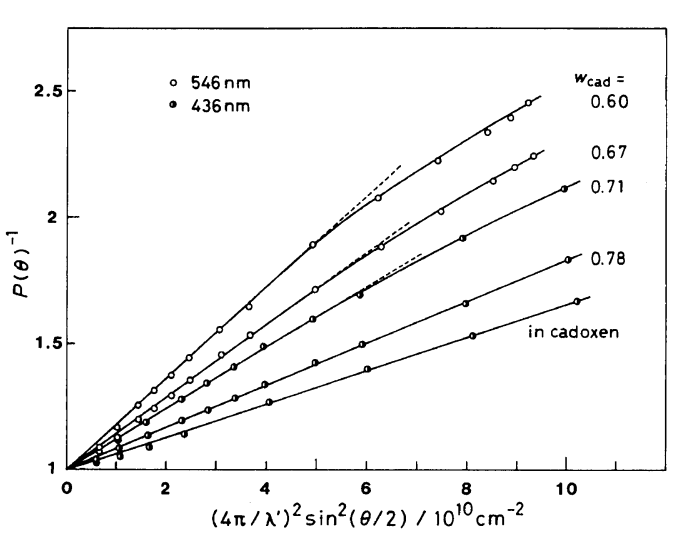

Figure A3. Angular dependence of $P(\theta)^{-1}$ for the xanthan sample in water-diluted cadoxen with different $w_{\text {cad }}$ at $25^{\circ} \mathrm{C}$.

water-diluted solution with $w_{\text {cad }}=0.10$ rapidly leveled off within $0.5 \mathrm{~h}$ after the preparation of the solution, following a marked, initial increase with time.

Figure A1 shows plots of $\left(\ln \eta_{\mathrm{r}}\right) / c$ vs. $c$ for water-diluted cadoxen solutions at $25^{\circ} \mathrm{C}$. The unusually large slope of the curve for $w_{\text {cad }}=$ 0.10 (the Huggins constant is 1.4) confirms the previous conclusion ${ }^{4}$ that a pronounced interchain association of $\mathrm{Na}$ salt xanthan takes place in a water-diluted cadoxen solution with this cadoxen composition.

Light scattering data for water-diluted ca- doxen solutions are shown in Figures A2 and A3. In the former, $A_{2}$ for $w_{\text {cad }}=0.60$ is seen to be almost zero, which indicates interchain association of xanthan chains at finite $c$.

The values of $M_{w},\left\langle S^{2}\right\rangle_{z}^{1 / 2}$, and $[\eta]$ in Figure 5 were evaluated from the intercepts and slopes of the indicated straight lines in Figures $\mathrm{A} 1-\mathrm{A} 3$.

\section{REFERENCES}

1. P. E. Jansson, L. Kenne, and B. Lindberg, Carbohydr. Res., 45, 275 (1975).

2. L. D. Melton, L. Mindt, D. A. Rees, and G. R. Sandersson, Carbohydr. Res., 46, 245 (1976).

3. T. Sato, T. Norisuye, and H. Fujita, Polym. J., 16, 341 (1984).

4. T. Sato, S. Kojima, T. Norisuye, and H. Fujita, Polym. J., 16, 423 (1984).

5. T. Sato, T. Norisuye, and H. Fujita, Macromolecules, 17, 2696 (1984).

6. K. Okuyama, S. Arnott, R. Moorhouse, M. D. Walkinshow, E. D. T. Atkins, and Ch. Wolf-Ullish, "Fiber Diffraction Methods," A. D. French and K. H. Gardner Ed., ACS Sym. Ser. No. 141, Am. Chem. Soc., Washington, D.C., 1980, p 411.

7. T. Sato, K. Sakurai, T. Norisuye, and H. Fujita, Polym. J., 15, 87 (1983).

8. I. T. Norton, D. M. Goodall, E. R. Morris, and D. A. Rees, J. Chem. Soc., Chem. Commun., 545 (1980).

9. I. T. Norton, D. M. Goodall, S. A. Frangou, E. R. Morris, and D. A. Rees, J. Mol. Biol., 175, 371 (1984). 